\title{
CORDIC: Funções hiperbólicas
}

\author{
Claudemir Aniz Antônio Giansante
}

\section{Resumo}

O COordinate Rotation DIgital Computer, abreviado por CORDIC, é um método numérico introduzido por Volder para calcular funções trigonométricas. Walther generalizou este algoritmo para calcular funções hiperbólicas, logarítmicas, exponenciais e raiz quadrada. Neste artigo, apresentaremos este método para as funções seno e cosseno hiperbólicos.

Palavras-chave: cosseno hiperbólico; seno hiperbólico; condição de convergência

\begin{abstract}
The Coordinate Rotation DIgital Computer, abbreviated by CORDIC, is a numerical method introduced by Volder to calculate trigonometric functions. Walther generalized this algorithm to calculate hyperbolic, logarithmic, exponential and square root functions. In this paper, we will present this method for the sine and cosine hyperbolic functions.
\end{abstract}

Keywords: hyperbolic cosine; hyperbolic sine; convergence condition

\section{Introdução}

De acordo com Sultan [7], as calculadoras utilizam o método CORDIC para calcular seno, cosseno e outras funções. Em 1959 Jack E. Volder [9] desenvolveu o algoritmo CORDIC para o cálculo em tempo real das funções trigonométricas sem o uso de muito hardware, e sugeriu que com pequenas modificações poderia se calcular outras funções elementares. Em 1971, J. S. Walther [10] generalizou este método para calcular funções hiperbólicas, exponenciais, logarítmicas e raiz quadrada. O CORDIC que aproxima as funções seno e cosseno hiperbólicos, consiste em realizar $n$ iterações em três equações, dadas por

$$
\left\{\begin{array}{l}
x_{k+1}=x_{k}+\delta_{k} y_{k} 2^{-\phi(k)} \\
y_{k+1}=y_{k}+\delta_{k} x_{k} 2^{-\phi(k)} \\
z_{k+1}=z_{k}-\delta_{k} \varepsilon_{k}
\end{array} .\right.
$$

Sendo $k \geq 1, \varepsilon_{k}=\tanh ^{-1}\left(2^{-\phi(k)}\right), x_{1}=\prod_{k=1}^{n} \cosh \varepsilon_{k}, y_{1}=0, z_{1}=\theta, \tanh ^{-1}$ a função inversa da tangente hiperbólica e $\delta_{k}=\operatorname{sgn}\left(z_{k}\right)=\left\{\begin{array}{l}1 \text { se } z_{k} \geq 0 \\ -1 \text { se } z_{k}<0\end{array}\right.$

A função $\phi: \mathbb{N}\{0\} \rightarrow \mathbb{N}\{0\}$ é 
definida pondo $\phi(k)=k-p$, onde $p$ é o maior inteiro tal que $3^{p+1}+2 p-1 \leq 2 k$. Uma condição para convergência é dada pelo teorema 1 , e o erro que se comete nas aproximações dependende do número de iterações. As adaptações feitas por Walther para garantir a convergência, e que são realizadas pela função $\phi$, são citadas em [10] com indicação sucinta de sua demonstração. O principal objetivo deste artigo é demonstrar em detalhes estas correções. Outros trabalhos utilizando CORDIC no cálculo das funções elementares podem ser encontradas em [2], [3], [5], [6] e [8] .

Este trabalho esta dividido em duas partes. A primeira contém um resumo das principais propriedades das funções hiperbólicas e um exemplo de $\operatorname{como}$ calcular $\cosh \theta$ e $\operatorname{senh} \theta$ usando rotações hiperbólicas. A segunda é dedicada à apresentação detalhada do algoritmo CORDIC com foco na demonstração das correções que garantem a sua convergência.

\section{Funções hiperbólicas}

Considere a hipérbole $H=\left\{(x, y) \in \mathbb{R} \mid x^{2}-y^{2}=1\right\}$. O ponto $P=(x, y) \in H$ define um setor hiperbólico $A O P$ e um ângulo hiperbólico $\widehat{A O P}$. Dizemos que o ângulo hiperbólico $\widehat{A O P}$ mede $\theta$ se a área do setor hiperbólico $A O P$ mede $\theta / 2$ unidades de área, Figura (1). Convenção:

i) Se o ponto $P$ estiver acima do eixo $x$, o ângulo terá medida positiva.

ii) Se o ponto $P$ estiver abaixo do eixo $x$, o ângulo terá medida negativa.

Com esta convenção os valores de $\theta$ estarão entre $-\infty$ e $+\infty$.

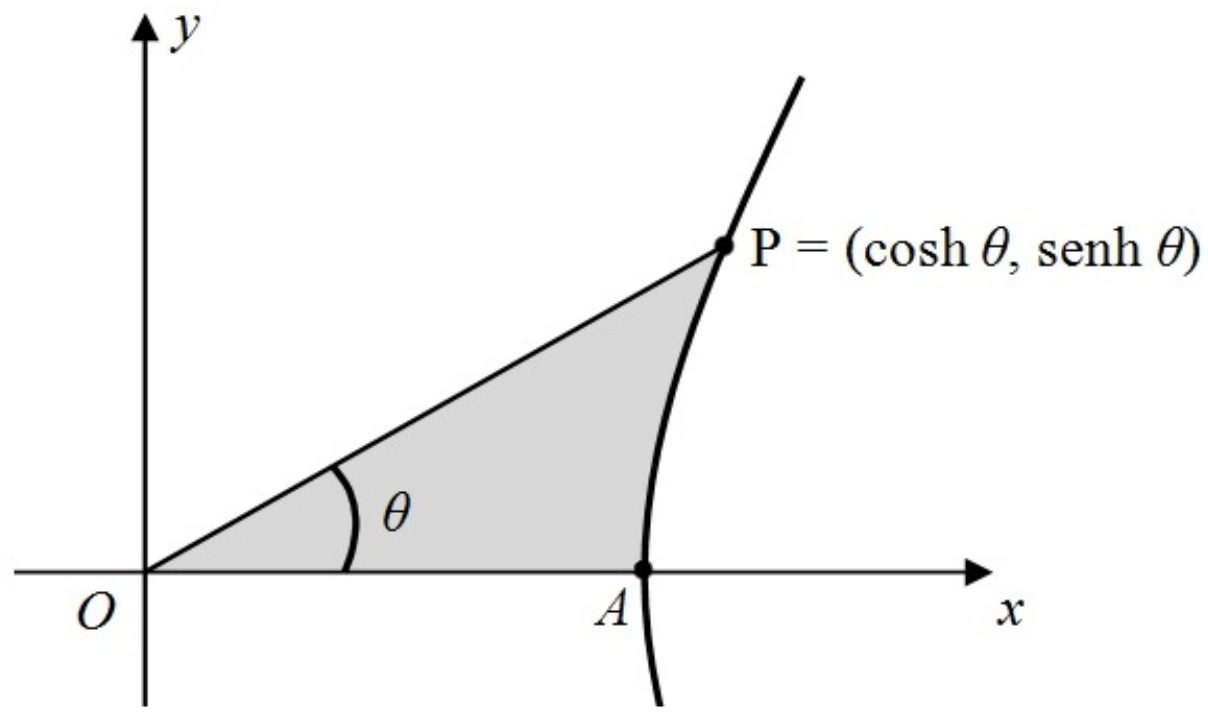

Figura 1: setor hiperbólico 
O ponto $P$ tem coordenadas $x=\cosh \theta$ e $y=\operatorname{senh} \theta$, cosseno e seno hiperbólico. Em [1], encontramse as seguintes igualdades:

iii) $\cosh \theta=\frac{e^{\theta}+e^{-\theta}}{2}$;

iv) $\operatorname{senh} \theta=\frac{e^{\theta}-e^{-\theta}}{2}$.

Usando as relações (iii) e (iv), podemos mostrar que :

v) $\cosh \left(\theta_{1} \pm \theta_{2}\right)=\cosh \theta_{1} \cosh \theta_{2} \pm \operatorname{senh} \theta_{1} \operatorname{senh} \theta_{2}$

vi) $\operatorname{senh}\left(\theta_{1} \pm \theta_{2}\right)=\operatorname{senh} \theta_{1} \cosh \theta_{2} \pm \cosh \theta_{1} \operatorname{senh} \theta_{2}$;

vii) A função cosseno hiperbólico é par e a função seno hiperbólico é ímpar.

Sendo $P_{1}=\left(x_{1}, y_{1}\right) \in H$, a rotação hiperbólica de um ângulo $\theta$ do vetor $\overrightarrow{O P_{1}}$ é o deslocamento do vetor de modo que sua extremidade permaneça em $H$ e percorra um ângulo hiperbólico $\theta$. $\mathrm{O}$ vetor $\overrightarrow{O P_{2}}$ obtido por esta rotação tem coordenadas $\left(x_{2}, y_{2}\right) \in H$ onde

$$
\left[\begin{array}{l}
x_{2} \\
y_{2}
\end{array}\right]=M_{\theta} \cdot\left[\begin{array}{l}
x_{1} \\
y_{1}
\end{array}\right] \text { e } M_{\theta}=\left[\begin{array}{cc}
\cosh \theta & \operatorname{senh} \theta \\
\operatorname{senh} \theta & \cosh \theta
\end{array}\right] \text {. }
$$

Observe que a rotação hiperbólica não tem o mesmo significado da rotação circular.

A função tangente hiperbólica é definida por $\tanh \theta=\frac{\operatorname{senh} \theta}{\cosh \theta}$ e vale a igualdade $\tanh \theta=\frac{e^{2 \theta}-1}{e^{2 \theta}+1}$. Os próximos resultados serão utilizados na última seção.

Lema 1. $\frac{1}{2} \tanh \theta \leq \tanh \left(\frac{\theta}{2}\right)$ para todo $\theta \geq 0$.

Demonstração. $\frac{1}{2} \tanh \theta \leq \tanh \left(\frac{\theta}{2}\right)$ se, e somente se, $\frac{1}{2} \cdot \frac{e^{2 \theta}-1}{e^{2 \theta}+1} \leq \frac{e^{\theta}-1}{e^{\theta}+1}$ se, e somente se, $\left(e^{2 \theta}-1\right)\left(e^{\theta}+1\right) \leq 2\left(e^{\theta}-1\right)\left(e^{2 \theta}+1\right)$ se, e somente se, $\left(e^{\theta}-1\right)^{3} \geq 0$. A última desigualdade é verdadeira para todo $\theta \geq 0$.

Lema 2. $\frac{1}{2^{n}} \leq \tanh \left(\frac{1}{2^{n-1}}\right)$ para todo natural $n \geq 1$.

Demonstração. A demonstração é feita por indução. Para $n=1$, a desigualdade é verdadeira, visto que, $\frac{1}{2} \leq \tanh (1) \approx 0.762$. Se $\frac{1}{2^{n}} \leq \tanh \left(\frac{1}{2^{n-1}}\right)$ para algum $n \geq 1$, então

$$
\frac{1}{2} \cdot \frac{1}{2^{n}} \leq \frac{1}{2} \cdot \tanh \left(\frac{1}{2^{n-1}}\right) \leq \tanh \left(\frac{1}{2} \cdot \frac{1}{2^{n-1}}\right) .
$$

Ou seja, $\frac{1}{2^{n+1}} \leq \tanh \left(\frac{1}{2^{n}}\right)$

A função tangente hiperbólica é estritamente crescente e sua inversa será denotada por $\tanh ^{-1}$. Pelo lema 2 , temos $\tanh ^{-1}\left(\frac{1}{2^{n}}\right) \leq \frac{1}{2^{n-1}}$, para todo $n \geq 1$. 


\section{Calculando cosseno e seno hiperbólico}

Dado $\theta$, suponha que queremos calcular $\cosh \theta$ e $\operatorname{senh} \theta$. A ideia é aproximar o ângulo $\theta$ por somas de ângulos $\pm \theta_{k}$ onde $\theta_{k}=\tanh ^{-1}\left(1 / 2^{k}\right)$ para números naturais $k \geq 1$. Ao se aproximar o ângulo $\theta$ pela soma $s_{n+1}$, os valores de $\cosh \theta$ e $\operatorname{senh} \theta$ são aproximados pelas coordenadas do vetor $\overrightarrow{O P_{n+1}}=\left(\cosh \left(s_{n+1}\right), \operatorname{senh}\left(s_{n+1}\right)\right)$ obtido do vetor $\overrightarrow{O P_{1}}=(1,0)$ pela rotação hiperbólica do ângulo $s_{n+1}$, isto é,

$$
\left[\begin{array}{c}
\cosh \left(s_{n+1}\right) \\
\operatorname{senh}\left(s_{n+1}\right)
\end{array}\right]=M_{ \pm \theta_{n}} \cdots M_{ \pm \theta_{1}} \cdot\left[\begin{array}{l}
1 \\
0
\end{array}\right]
$$

Observe que,

$$
M_{ \pm \theta_{k}}=\left[\begin{array}{cc}
\cosh \left( \pm \theta_{k}\right) & \operatorname{senh}\left( \pm \theta_{k}\right) \\
\operatorname{senh}\left( \pm \theta_{k}\right) & \cosh \left( \pm \theta_{k}\right)
\end{array}\right]=\cosh \theta_{k} \cdot\left[\begin{array}{cc}
1 & \tanh \left( \pm \theta_{k}\right) \\
\tanh \left( \pm \theta_{k}\right) & 1
\end{array}\right]
$$

Para exemplificar, calculemos $\cosh \theta$ e $\operatorname{senh} \theta$ para $\theta=0.61$, Figura (2). O ângulo $\theta=0.61$ é aproximado por

$$
\begin{aligned}
s_{5} & =\theta_{1}+\theta_{2}-\theta_{3}-\theta_{4} \\
& =0.549306144+0.255412811-0.125657214-0.062581571 \\
& =0.616480170
\end{aligned}
$$

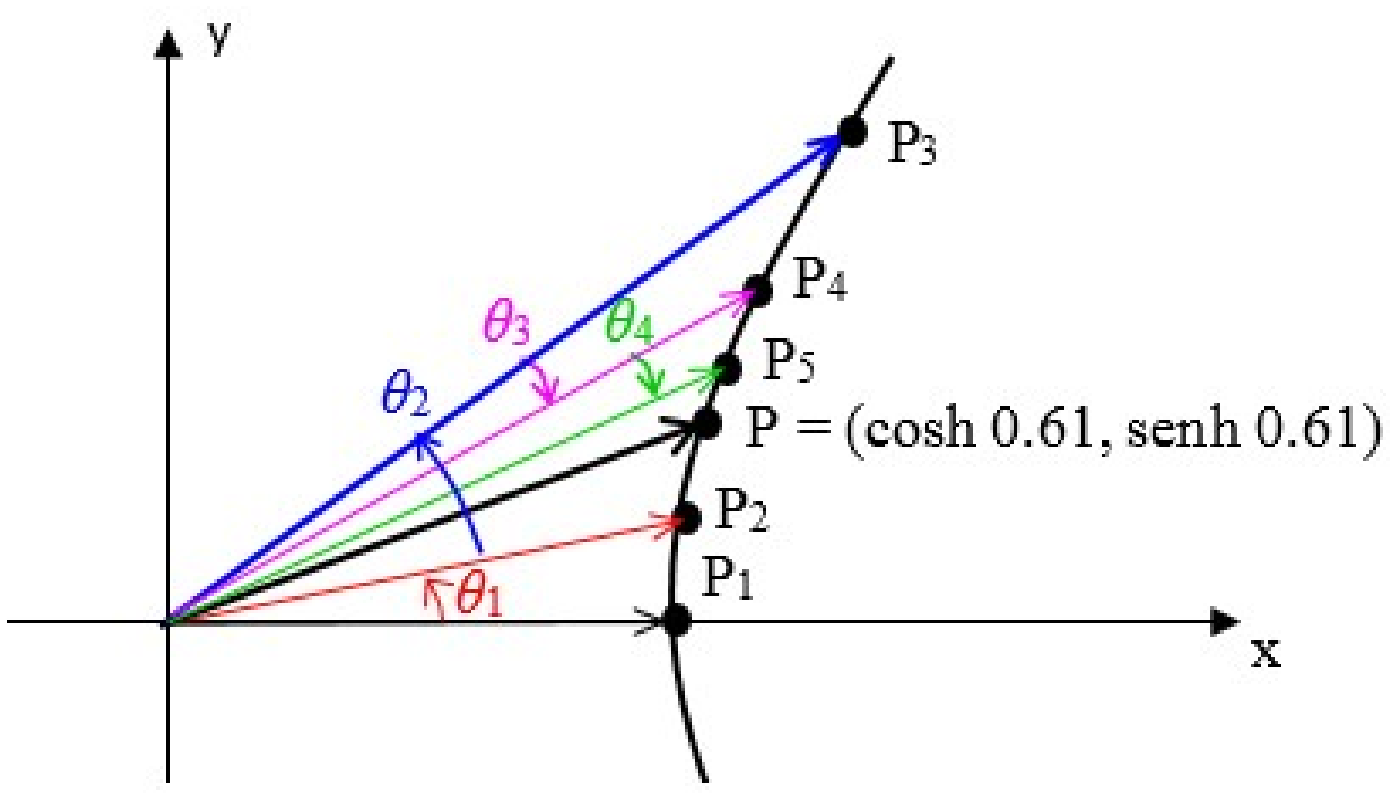

Figura 2: sequência de aproximações 
Então,

$$
\left[\begin{array}{c}
\cosh \left(s_{5}\right) \\
\operatorname{senh}\left(s_{5}\right)
\end{array}\right]=K \cdot\left[\begin{array}{cc}
1 & -\frac{1}{16} \\
-\frac{1}{16} & 1
\end{array}\right]\left[\begin{array}{cc}
1 & -\frac{1}{8} \\
-\frac{1}{8} & 1
\end{array}\right]\left[\begin{array}{cc}
1 & \frac{1}{4} \\
\frac{1}{4} & 1
\end{array}\right]\left[\begin{array}{cc}
1 & \frac{1}{2} \\
\frac{1}{2} & 1
\end{array}\right] \cdot\left[\begin{array}{l}
1 \\
0
\end{array}\right]
$$

sendo $K=\cosh \theta_{4} \cdot \cosh \theta_{3} \cdot \cosh \theta_{2} \cdot \cosh \theta_{1}=1.204351713$.

Efetuando os produtos, obtemos

$$
\left[\begin{array}{c}
\cosh \left(s_{5}\right) \\
\operatorname{senh}\left(s_{5}\right)
\end{array}\right]=1.204351713 \cdot\left[\begin{array}{l}
0.993164062 \\
0.544921875
\end{array}\right]=\left[\begin{array}{l}
1.196118839 \\
0.656277593
\end{array}\right] .
$$

Logo, $\cosh 0.61 \approx 1.196118839$ e senh $0.61 \approx 0.656277593$.

Infelizmente, não podemos garantir que somas de ângulos $\pm \theta_{k}$ convirjam para qualquer valor de $\theta$. A próxima seção fornece uma condição para que isto ocorra.

\section{Condição de convergência}

A garantia de convergência do algoritmo CORDIC depende do próximo teorema, demonstrado em $[6]$.

Teorema 1. (Teorema de Convergência) Suponha que $\varepsilon_{1} \geq \varepsilon_{2} \geq \cdots \geq \varepsilon_{n}>0$ é uma sequência finita de números reais tal que

$$
\varepsilon_{k} \leq \varepsilon_{n}+\sum_{j=k+1}^{n} \varepsilon_{j}, \quad \text { para } 1 \leq k<n,
$$

e suponha que $r$ é um número real tal que

$$
|r| \leq \sum_{j=1}^{n} \varepsilon_{j}
$$

Se $s_{1}=0$ e $s_{k+1}=s_{k}+\delta_{k} \varepsilon_{k}$ para $1 \leq k \leq n$, onde

$$
\delta_{k}=\operatorname{sgn}\left(r-s_{k}\right)=\left\{\begin{array}{l}
1, \text { se } r \geq s_{k} \\
-1, \text { se } r<s_{k}
\end{array}\right.
$$

então

$$
\left|r-s_{k}\right| \leq \varepsilon_{n}+\sum_{j=k}^{n} \varepsilon_{j}, \quad \text { para } 1 \leq k \leq n,
$$

em particular $\left|r-s_{n+1}\right| \leq \varepsilon_{n}$.

A desigualdade (1) é a condição de convergência, e a desigualdade (2) fornece o raio de convergência. A sequência de números reais positivos $\theta_{k}=\tanh ^{-1}\left(2^{-k}\right)$, com $1 \leq k \leq 13$, é decrescente mas não satisfaz a condição de convergência, Tabela (1). 


\begin{tabular}{|c|c|c|c|c|c|}
\hline & $\theta_{k}$ & $\theta_{13}+\sum_{j=k+1}^{13} \theta_{j}$ & & $\theta_{k}$ & $\theta_{13}+\sum_{j=k+1}^{13} \theta_{j}$ \\
\hline 1 & 0.549306144 & 0.506163226 & 8 & 0.003906270 & 0.003906252 \\
\hline 2 & 0.255412811 & 0.250750415 & 9 & 0.001953127 & 0.001953125 \\
\hline 3 & 0.125657214 & 0.125093201 & 10 & 0.000976563 & 0.000976562 \\
\hline 4 & 0.062581571 & 0.062511630 & 11 & 0.000488281 & 0.000488281 \\
\hline 5 & 0.031260178 & 0.031251452 & 12 & 0.000244141 & 0.000244140 \\
\hline 6 & 0.015626271 & 0.015625181 & 13 & 0.000122070 & \\
\hline 7 & 0.007812659 & 0.007812522 & & & \\
\hline
\end{tabular}

Tabela 1: sequência $\theta_{k}$, com $1 \leq k \leq 13$, seguida das somas da desigualdade (1)

Duplicando as entradas quatro e treze da Tabela (1), obtemos a sequência $\varepsilon_{k}$, para $1 \leq k \leq 15$, Tabela (2).

\begin{tabular}{|c|c|c|c|c|c|}
\hline & $\varepsilon_{k}$ & $\varepsilon_{15}+\sum_{j=k+1}^{15} \varepsilon_{j}$ & & $\varepsilon_{k}$ & $\varepsilon_{15}+\sum_{j=k+1}^{15} \varepsilon_{j}$ \\
\hline 1 & 0.549306144 & 0.568866867 & 9 & 0.003906270 & 0.004028322 \\
\hline 2 & 0.255412811 & 0.313454056 & 10 & 0.001953127 & 0.002075195 \\
\hline 3 & 0.125657214 & 0.187796842 & 11 & 0.000976563 & 0.001098632 \\
\hline $4^{*}$ & 0.062581571 & 0.125215271 & 12 & 0.000488281 & 0.000610351 \\
\hline $5^{*}$ & 0.062581571 & 0.062633700 & 13 & 0.000244141 & 0.000366210 \\
\hline 6 & 0.031260178 & 0.031373522 & $14^{*}$ & 0.000122070 & 0.000244140 \\
\hline 7 & 0.015626271 & 0.015747251 & $15^{*}$ & 0.000122070 & \\
\hline 8 & 0.007812659 & 0.007934592 & & & \\
\hline
\end{tabular}

Tabela 2: sequência obtida da Tabela (1), duplicando as entradas quatro e quatorze.

Observando a Tabela (2), podemos concluir que $\varepsilon_{k} \leq \varepsilon_{15}+\sum_{j=k+1}^{15} \varepsilon_{j}$ para todo $1 \leq k<15$, isto é, a sequência $\varepsilon_{k}$ satisfaz a condição de convergência. Em [10], Walther pontuou que os índices que devem ser repetidos pertencem ao conjunto $\{4,13,40,121, \ldots, i, 3 i+1, \ldots\}$. Na próxima seção apresentaremos a demonstração deste resultado.

\section{Correções da sequência}

Esta seção contém a parte principal deste trabalho. Demonstramos em detalhes as correções feitas na sequência $\theta_{k}$ para garantir a condição de convergência.

Lema 3. $2^{-3 n+3}-2^{-3 n+2} \geq 2^{-5 n+5}-2^{-5 n+3}$ para todo natural $n \geq 2$.

Demonstração. Vamos demonstrar a desigualdade utilizando indução matemática. Para $n=2$, a desigualdade $2^{-3}-2^{-4} \geq 2^{-5}-2^{-7}$ é verdadeira, pois é equivalente a $\frac{8}{128} \geq \frac{3}{128}$. Suponha que $2^{-3 n+3}-2^{-3 n+2} \geq 2^{-5 n+5}-2^{-5 n+3}$ é verdadeira para $n \geq 2$. Como $2^{-3} \geq 2^{-5}$, então

$$
2^{-3}\left(2^{-3 n+3}-2^{-3 n+2}\right) \geq 2^{-5}\left(2^{-5 n+5}-2^{-5 n+3}\right) .
$$

Ou seja, $2^{-3(n+1)+3}-2^{-3(n+1)+2} \geq 2^{-5(n+1)+5}-2^{-5(n+1)+3}$. 
Em [4], pág. 525, encontra-se a igualdade $\theta_{k}=\tanh ^{-1}\left(2^{-k}\right)=\frac{1}{2} \ln \left(\frac{1+2^{-k}}{1-2^{-k}}\right)$ para todo $k \geq 1$, que será utilizada nos próximos resultados.

Lema 4. $2 \theta_{n}+\theta_{3(n-1)+1}-\theta_{n-1} \geq 0$ para todo natural $n \geq 2$.

Demonstração. As desigualdades abaixo são equivalentes.

i) $2 \theta_{n}+\theta_{3(n-1)+1}-\theta_{n-1} \geq 0$;

ii) $\frac{1}{2} \ln \left[\left(\frac{1+2^{-n}}{1-2^{-n}}\right)^{2} \cdot\left(\frac{1-2^{-(n-1)}}{1+2^{-(n-1)}}\right) \cdot\left(\frac{1+2^{-(3 n-2)}}{1-2^{-(3 n-2)}}\right)\right] \geq 0$;

iii) $\left(1+2^{-n}\right)^{2} \cdot\left(1-2^{-n+1}\right) \cdot\left(1+2^{-3 n+2}\right) \geq\left(1-2^{-n}\right)^{2} \cdot\left(1+2^{-n+1}\right) \cdot\left(1-2^{-3 n+2}\right)$;

iv) $2^{-3 n+3}-2^{-5 n+5}+2^{-5 n+3}-2^{-3 n+2} \geq 0$.

Pelo lema 3, a desigualdade (iv) é verdadeira.

Lema 5. $2^{-3 m+2}-2^{-3 m-1}-2^{-3 m+1} \geq-2^{-5 m+2}+2^{-5 m+4}$ para todo natural $m \geq 2$.

Demonstração. Vamos demonstrar a desigualdade utilizando indução matemática. Para $m=2$, a desigualdade $2^{-4}-2^{-7}-2^{-5} \geq-2^{-8}+2^{-6}$ é verdadeira, pois é equivalente a $\frac{6}{256} \geq \frac{3}{256}$. Suponha que $2^{-3 m+2}-2^{-3 m-1}-2^{-3 m+1} \geq-2^{-5 m+2}+2^{-5 m+4}$ é verdadeira para $m \geq 2$. Como $2^{-3} \geq 2^{-5}$, então $2^{-3}\left(2^{-3 m+2}-2^{-3 m-1}-2^{-3 m+1}\right) \geq 2^{-5}\left(-2^{-5 m+2}+2^{-5 m+4}\right)$. Portanto,

$$
2^{-3(m+1)+2}-2^{-3(m+1)-1}-2^{-3(m+1)+1} \geq-2^{-5(m+1)+2}+2^{-5(m+1)+4} .
$$

Lema 6. $2 \theta_{m}-\theta_{3 m+1}-\theta_{m-1}+\theta_{3(m-1)+1} \geq 0$ para todo natural $m \geq 2$.

Demonstração. As desigualdades abaixo são equivalentes.

i) $2 \theta_{m}-\theta_{3 m+1}-\theta_{m-1}+\theta_{3(m-1)+1} \geq 0$;

ii) $\frac{1}{2} \ln \left[\left(\frac{1+2^{-m}}{1-2^{-m}}\right)^{2}\left(\frac{1-2^{-(m-1)}}{1+2^{-(m-1)}}\right)\left(\frac{1+2^{-(3 m-2)}}{1-2^{-(3 m-2)}}\right)\left(\frac{1-2^{-(3 m+1)}}{1+2^{-(3 m+1)}}\right)\right] \geq 0$;

iii) $M_{1} \geq M_{2}$, sendo $M_{1}=\left(1+2^{-m}\right)^{2}\left(1-2^{-m+1}\right)\left(1+2^{-3 m+2}\right)\left(1-2^{-3 m-1}\right)$ e

$$
M_{2}=\left(1-2^{-m}\right)^{2}\left(1+2^{-m+1}\right)\left(1-2^{-3 m+2}\right)\left(1+2^{-3 m-1}\right) ;
$$

iv) $S_{1}+S_{2} \geq 0$, onde $S_{1}=2^{-5 m+1}-2^{-5 m-1}+2^{-9 m+2}$ e

$$
S_{2}=2^{-3 m+2}-2^{-3 m-1}-2^{-3 m+1}+2^{-5 m+2}-2^{-5 m+4} .
$$


Como $S_{1} \geq 0$ para todo $m \geq 2$ e pelo lema $5, S_{2} \geq 0$, o resultado segue.

Lema 7. Se $\theta_{n-k} \leq \theta_{n}+\left(\sum_{j=(n-k)+1}^{n} \theta_{j}\right)+\theta_{3(n-k)+1}$ para $1 \leq k<n-1$ e $n \geq 3$, então

$$
\theta_{n-(k+1)} \leq \theta_{n}+\left(\sum_{j=(n-(k+1))+1}^{n} \theta_{j}\right)+\theta_{3(n-(k+1))+1} .
$$

Demonstração. As desigualdades abaixo são equivalentes:

i) $\theta_{n-(k+1)} \leq \theta_{n}+\sum_{j=n-k}^{n} \theta_{j}+\theta_{3(n-k-1)+1}$;

ii) $\theta_{n}+\sum_{j=n-k}^{n} \theta_{j}-\theta_{n-(k+1)}+\theta_{3(n-k-1)+1} \geq 0$;

iii) $N_{1}+N_{2}+N_{3}+N_{4} \geq 0$, onde $N_{1}=\theta_{n}+\left(\sum_{j=(n-k)+1}^{n} \theta_{j}\right)+\theta_{n-k}$,

$$
N_{2}=\theta_{n-k}-\theta_{n-k}, N_{3}=\theta_{3(n-k)+1}-\theta_{3(n-k)+1} \text { e } N_{4}=-\theta_{n-k-1}+\theta_{3(n-k-1)+1} \text {. }
$$

iv) $P_{1}+P_{2} \geq 0$, sendo $P_{1}=\theta_{n}+\left(\sum_{j=(n-k)+1}^{n} \theta_{j}\right)+\theta_{3(n-k)+1}-\theta_{n-k}$ e

$$
P_{2}=2 \theta_{n-k}-\theta_{3(n-k)+1}-\theta_{(n-k)-1}+\theta_{3((n-k)-1))+1} \text {. }
$$

Por hipótese $P_{1} \geq 0$ e pelo lema $6, P_{2} \geq 0$.

Teorema 2. Seja $\theta_{k}=\tanh ^{-1}\left(2^{-k}\right)$ para $k \geq 1$, então

$$
D_{k}: \theta_{n-k} \leq \theta_{n}+\left(\sum_{j=(n-k)+1}^{n} \theta_{j}\right)+\theta_{3(n-k)+1}, \text { para } 1 \leq k<n \text { e } n \geq 2 .
$$

Demonstração. A desigualdade $D_{k}$ é verdadeira para $k=1$, pois neste caso

$$
D_{1}: \theta_{n-1} \leq \theta_{n}+\theta_{n}+\theta_{3(n-1)+1},
$$

que é válida para todo $n \geq 2$, pelo lema 4 . Se $D_{k}$ é verdadeira para algum $1 \leq k<n-1$ e $n \geq 3$, então pelo lema $7, D_{k+1}$ é verdadeira.

Como consequência do teorema 2 , a sequência obtida repetindo os termos $\theta_{k}$ para $k$ pertencente ao conjunto $\{4,13,40,121, \ldots, i, 3 i+1, \ldots\}$ satisfaz a condição de convergência. A seguir apresentamos algumas desigualdades, decorrentes da desigualdade (3).

i) $\theta_{1} \leq \theta_{4}+\left(\theta_{2}+\theta_{3}+\theta_{4}\right)+\theta_{4}$

ii) $\theta_{2} \leq \theta_{4}+\left(\theta_{3}+\theta_{4}\right)+\theta_{7} \leq \theta_{4}+\left(\theta_{3}+\theta_{4}\right)+\theta_{4}$; 
iii) $\theta_{3} \leq \theta_{4}+\left(\theta_{4}\right)+\theta_{10} \leq \theta_{4}+\left(\theta_{4}\right)+\theta_{4}$;

iv) $\theta_{4} \leq \theta_{13}+\left(\theta_{5}+\cdots+\theta_{13}\right)+\theta_{13}$;

v) $\theta_{5} \leq \theta_{13}+\left(\theta_{6}+\cdots+\theta_{13}\right)+\theta_{16} \leq \theta_{13}+\left(\theta_{6}+\cdots+\theta_{13}\right)+\theta_{13}$;

vi) $\theta_{6} \leq \theta_{13}+\left(\theta_{7}+\cdots+\theta_{13}\right)+\theta_{19} \leq \theta_{13}+\left(\theta_{7}+\cdots+\theta_{13}\right)+\theta_{13}$;

vii) $\theta_{13} \leq \theta_{40}+\left(\theta_{14}+\cdots+\theta_{40}\right)+\theta_{40}$;

viii) $\theta_{14} \leq \theta_{40}+\left(\theta_{15}+\cdots+\theta_{40}\right)+\theta_{43} \leq \theta_{40}+\left(\theta_{15}+\cdots+\theta_{40}\right)+\theta_{40}$.

As desigualdades de i) até viii) são para $k$ em $\{4,13,40\}$.

Em [5], pp139, define-se a função $\phi: \mathbb{N}\{0\} \rightarrow \mathbb{N}\{0\}$ pondo $\phi(k)=k-p$, onde $p$ é o maior inteiro tal que $3^{p+1}+2 p-1 \leq 2 k$. Para exemplificar, calculemos alguns valores de $\phi: \phi(43)=43-3=40$, $\phi(42)=42-2=40, \phi(41)=41-2=39, \phi(15)=15-2=13, \phi(14)=14-1=13$, $\phi(13)=13-1=12, \phi(5)=5-1=4, \phi(4)=4-0=4, \phi(3)=3-0=3$. Utilizando a função $\phi$, a sequência obtida com as repetições de $\theta_{k}$ pode ser escrita por $\varepsilon_{k}=\theta_{\phi(k)}$. Note que,

$\left(\varepsilon_{1}, \varepsilon_{2}, \varepsilon_{3}, \varepsilon_{4}, \varepsilon_{5}, \ldots, \varepsilon_{13}, \varepsilon_{14}, \varepsilon_{15}, \ldots, \varepsilon_{41}, \varepsilon_{42}, \varepsilon_{43}\right)=\left(\theta_{1}, \theta_{2}, \theta_{3}, \theta_{4}, \theta_{4}, \ldots, \theta_{12}, \theta_{13}, \theta_{13}, \ldots, \theta_{39}, \theta_{40}, \theta_{40}\right)$.

Para contornar o problema de qual $\theta_{k}$ deve ser repetido, Eklund [2] decide repetir todos os $\theta_{k}$ para $k \geq 2$. Neste caso, a demonstração do critério de convergência é mais simples, porém o algoritmo fica mais lento, sendo necessário um número maior de iterações para obter a mesma precisão usando a sequência $\varepsilon_{k}$.

\section{Algoritmo CORDIC}

Considere a matriz

$$
M_{ \pm \varepsilon_{k}}^{*}=\left[\begin{array}{cc}
1 & \tanh \left( \pm \varepsilon_{k}\right) \\
\tanh \left( \pm \varepsilon_{k}\right) & 1
\end{array}\right]=\left[\begin{array}{cc}
1 & \pm 2^{-\phi(k)} \\
\pm 2^{-\phi(k)} & 1
\end{array}\right] .
$$

Observe que,

$$
M_{ \pm \varepsilon_{k}}^{*} \cdot\left[\begin{array}{c}
x_{1} \\
y_{1}
\end{array}\right]=\left[\begin{array}{c}
x_{1} \pm y_{1} \cdot 2^{-\phi(k)} \\
y_{1} \pm x_{1} \cdot 2^{-\phi(k)}
\end{array}\right]
$$

Se $s_{n+1}=\sum_{k=1}^{n} \pm \varepsilon_{k}$, então

$$
\left[\begin{array}{c}
\cosh \left(s_{n+1}\right) \\
\operatorname{senh}\left(s_{n+1}\right)
\end{array}\right]=M_{ \pm \varepsilon_{n}}^{*} \cdots M_{ \pm \varepsilon_{1}}^{*} \cdot\left[\begin{array}{c}
K \\
0
\end{array}\right] \text { onde } K=\cosh \varepsilon_{n} \cdots \cosh \varepsilon_{1} .
$$

Reescrevendo a Equação (4) e adicionando a sequência que aproxima o ângulo $\theta$, obtemos as equações CORDIC para calcular seno e cosseno hiperbólico,

$$
\left\{\begin{array}{l}
x_{k+1}=x_{k}+\delta_{k} y_{k} 2^{-\phi(k)} \\
y_{k+1}=y_{k}+\delta_{k} x_{k} 2^{-\phi(k)} \\
z_{k+1}=z_{k}-\delta_{k} \varepsilon_{k}
\end{array} .\right.
$$


Aqui, $k \geq 1, \varepsilon_{k}=\tanh ^{-1}\left(2^{-\phi(k)}\right), x_{1}=\prod_{k=1}^{n} \cosh \varepsilon_{k}, y_{1}=0, z_{1}=\theta \mathrm{e}$

$$
\delta_{k}=\operatorname{sgn}\left(z_{k}\right)=\left\{\begin{array}{l}
1 \text { se } z_{k} \geq 0 \\
-1 \text { se } z_{k}<0
\end{array} .\right.
$$

A soma dos elementos da Tabela (2) é $\sum_{j=1}^{15} \varepsilon_{j}=1.118050941$. Portanto, para $n \geq 15$ os valores de $|\theta| \leq 1.11$ pertencem ao intervalo de convergência.

Teorema 3. Se $|\theta| \leq 1.11$, então $\left|\cosh \theta-x_{n+1}\right|<\frac{1}{2^{\phi(n)-2}} e\left|\operatorname{senh} \theta-y_{n+1}\right|<\frac{1}{2^{\phi(n)-2}}$.

Demonstração. Note que, $x_{n+1}=\cosh \left(s_{n+1}\right)$ e $y_{n+1}=\operatorname{senh}\left(s_{n+1}\right)$. Aplicando o teorema do valor médio ([4], pág. 232), a função cosseno hiperbólico, existe um número $c$ entre $\theta$ e $s_{n+1}$ tal que

$$
\frac{\cosh \theta-\cosh \left(s_{n+1}\right)}{\theta-s_{n+1}}=\cosh ^{\prime} c=\operatorname{senh} c .
$$

Pelo teorema $1,\left|\theta-s_{n+1}\right| \leq \varepsilon_{n}=\tanh ^{-1}\left(2^{-\phi(n)}\right)$, e pelo lema $2, \tanh ^{-1}\left(2^{-\phi(n)}\right) \leq 1 / 2^{\phi(n)-1}$.

Segue que,

$$
\left|\cosh \theta-x_{n+1}\right| \leq\left|\theta-s_{n+1}\right| \cdot|\operatorname{senh} c| \leq \frac{1}{2^{\phi(n)-1}} \cdot|\operatorname{senh} c|
$$

Como a função seno hiperbólico é estritamente crescente e $|c| \leq 1.2$, então $|\operatorname{senh} c|<2$.

Portanto,

$$
\left|\cosh \theta-x_{n+1}\right|<\frac{1}{2^{\phi(n)-1}} \cdot 2=\frac{1}{2^{\phi(n)-2}} .
$$

Analogamente,

$$
\left|\operatorname{senh} \theta-y_{n+1}\right| \leq\left|\theta-s_{n+1}\right| \cdot|\cosh c|<\frac{1}{2^{\phi(n)-1}} \cdot 2=\frac{1}{2^{\phi(n)-2}}
$$

O teorema 3 fornece um limitante superior para o erro cometido ao se aproximar $\operatorname{senh} \theta$ e $\cosh \theta$ por $\operatorname{senh}\left(s_{n+1}\right)$ e $\cosh \left(s_{n+1}\right)$. Para $n=43$, o erro é menor que $\frac{1}{2^{\phi(43)-2}}=\frac{1}{2^{38}} \approx 3.638 \cdot 10^{-12}$, portanto a precisão é de 10 casas decimais.

\section{Referências}

[1] Carvalho, Sonia Pinto de. As Funções Hiperbólicas. Disponível em: http://docplayer.com.br/5125774-Sonia-pinto-de-carvalho.html, acessado em 14/12/2017.

[2] Eklund, N. CORDIC: Elemetary Function Computation Using Recursive Sequences. Eletronic Proceeding of the ICTCM, C027, 1998. 
[3] Giansante, Antônio Cézare de Araújo. Algoritmo CORDIC no cálculo das funções elementares. Dissertação de Mestrado - Profmat, Universidade Federal de Mato Grosso do Sul, Campo Grande, 2014.

[4] Leithold, Louis. O Cálculo com Geometria Analítica. Volume 1, Terceira Edição, Editora Harbra, 1994.

[5] Muller, Jean-Michel. Elementary Functions: Algorithms and Implementation. Second Edition, Birkhäuser Boston, 2006.

[6] Schelin, Charles W. Calculator Function Approximation. The American Mathematical Monthly, Vol. 90, No. 5, 317- 325, 1983.

[7] Sultan, Alan. CORDIC: How Hand Calculators Calculate. The College Mathematics Journal, 87-92, 2009.

[8] Underwood, J. and Edwards, B. How do calculators calculate trigonometric functions? Disponível em: https://people.clas.ufl.edu/bruceedwards/files/paper.pdf, acessado em 22/12/2017.

[9] Volder, J. E. The CORDIC Trigonometric Computing Technique. IRE Trans. Computers, EC-8, 330-334, 1959.

[10] Walther, J. S. A Unified Algorithm for Elementary Functions. Joint Computer Conference Proceedings, Springer Vol. 38, 379-385, 1971.

Recebido: 12/01/2018

Claudemir Aniz

UFMS - Campo Grande - MS $<$ claudemir.aniz@ufms.br>

Antônio Giansante UNEMAT - Tangará da Serra - MT

<profcabi@yahoo.com.br> 